\title{
Linear Iterative Demodulation Algorithm for MIMO Systems with Large Number of Antennas
}

\author{
D.Yu. Pankratov ${ }^{1}$, \\ Department of Systems and Networks of Radio Communications \\ and Radio Broadcasting
}

\author{
A.G. Stepanova Department of Theory of Electrical Circuits \\ Moscow Technical University of Communications and \\ Informatics \\ 12dpankr@mail.ru; ${ }^{2}$ ag.otc@ rambler.ru
}

\begin{abstract}
The use of Massive MIMO technology (i.e. MIMO technology with large number of antennas) is planned in 5G networks for significant capacity increasing. However, on the way to practical application of Massive MIMO technology in 5G systems, there are many problems, in particular, problems of digital signal processing algorithms synthesis on the receiving side, therefore, the developing task of demodulation algorithms with good quality characteristics and low computational complexity is very important.
\end{abstract}

In this paper we discuss linear demodulation algorithms for MIMO systems: Zero-Forcing (ZF) algorithm, minimum mean square error (MMSE) algorithm and Chebyshev iterative algorithms. For these algorithms BER performance characteristics are obtained.

Key words: 5G, Massive MIMO, demodulation algorithms, Chebyshev iterative algorithms, BER performance.

To significantly increase the capacity of modern radio communication systems, Multiple Input Multiple Output technology (MIMO) is widely used $[1 ; 2]$, which uses multiple transmitting and multiple receiving antennas.

In $5 \mathrm{G}$ networks, in order to increase the capacity, it is planned to use Massive MIMO technology (i.e. MIMO technology with a large number of antennas). This technology allows to obtain high spectral efficiency and high energy efficiency of the communication system. However, on the way to practical application of Massive MIMO technology in $5 \mathrm{G}$ systems, there are many problems, in particular, the problem of digital signal processing algorithms synthesis, therefore, the task of demodulation algorithms developing with good quality characteristics and low computational complexity is very important.

In this paper we discuss linear demodulation algorithms for MIMO systems: Zero-Forcing (ZF) algorithm, minimum mean square error (MMSE) algorithm and the Chebyshev iterative algorithms. The noise immunity curves of these algorithms for the MIMO system with 4 transmitting and 4 receiving antennas were obtained (bit error ratio (BER) versus signal-to-noise ratio (SNR)).

Figure 1 shows the MIMO block diagram. The model of the signal at the input of the demodulator is the following [2]:

$$
\boldsymbol{y}=\boldsymbol{H} \boldsymbol{s}+\boldsymbol{n},
$$

where $\mathbf{y}$ is received signals vector of $M \times 1$ dimension; $\boldsymbol{H}$ is complex matrix of MIMO radio channel of $M \times M$ dimension; $s$ is vector of transmitted information symbols of $M \times 1$ dimension; $\boldsymbol{n}$ is Gaussian random vector of noise of $\boldsymbol{M} \times 1$ dimension. Elements $\boldsymbol{h} \boldsymbol{i j}$ of MIMO channel matrix $\boldsymbol{H}$ represent the complex transmission coefficients from the $j$-th transmitting antenna to the $i$-th receiving antenna.

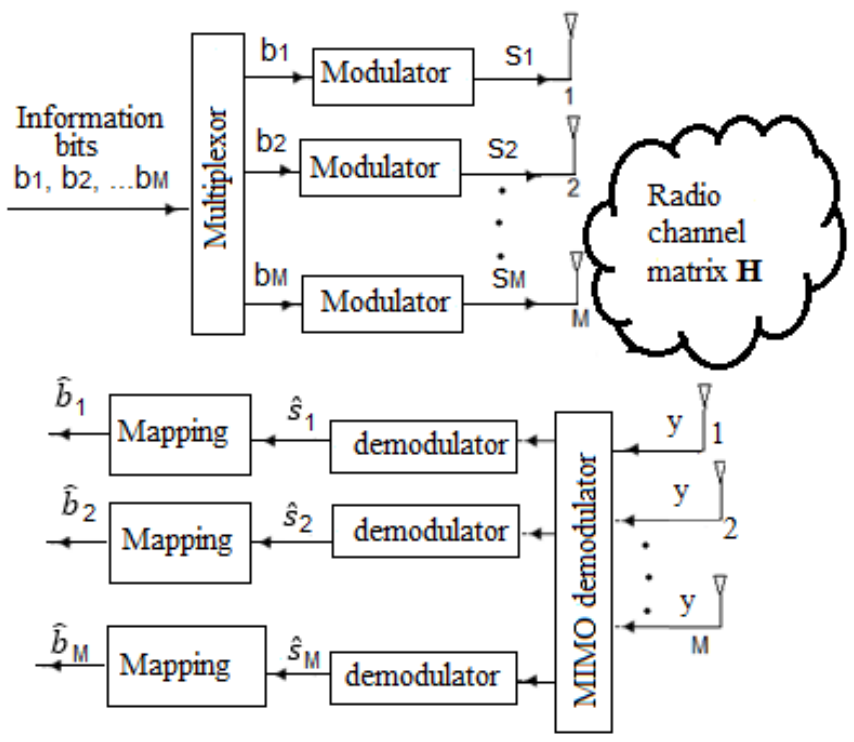

Fig. 1. MIMO system block diagram

The algorithm for MIMO system simulation has several steps:

- generation of bit vector $\mathbf{b}$ and information symbol vector $\mathbf{S}$ for all transmitting antennas, generation of MIMO channel matrix $\boldsymbol{H}$, consisting of complex coefficients hij;

- generation of complex Gaussian noise vector $\boldsymbol{n}$;

- $\quad$ obtaining of signal and noise mixture $\mathbf{y}$;

- demodulation of signal $\mathbf{y}$ - obtaining of information symbol vector estimation $\hat{\mathbf{S}}$ and corresponding vector $\hat{\mathbf{b}}$; 
- comparing of $\hat{\mathbf{b}}$ и $\mathbf{b}$ vectors and errors detection;

- bit error ratio (BER) calculation for given signalto-noise ratio (SNR).

These steps are performed for a given number of experiments and SNR values to achieve average BER performance for different SNR values. A detailed description of this algorithm can be found in paper [3].

In MIMO systems ZF (Zero Forcing) algorithm can be used for demodulation. The ZF estimation of information symbols can be found as follows $[2 ; 4]$ :

$$
\hat{\mathbf{s}}^{Z F}=\arg \min _{s \in C^{l}}\|\mathbf{y}-\mathbf{H s}\|^{2}=\left(\mathbf{H}^{\prime} \mathbf{H}\right)^{-1} \mathbf{H}^{\prime} \mathbf{y}
$$

where $C^{I}$ is $I$-dimensional continuous complex space; $\hat{\mathbf{S}}$ is estimation of received information symbol vector $\mathbf{S} ; \boldsymbol{y}$ is received signals vector of $M \times 1$ dimension; $\mathbf{H}$ is complex matrix of MIMO radio channel of $M \times M$ dimension; $\left(\mathbf{H}^{\prime} \mathbf{H}\right)^{-1} \mathbf{H}^{\prime}$ is pseudoinverse matrix with respect to the channel matrix $\mathbf{H} ; \mathbf{H}^{\prime}$ is Hermitian conjugate matrix with respect to the channel matrix $\mathbf{H}$. From (2) it can be seen that Zero Forcing algorithm of demodulation does not take into account the presence of observation noise $\mathbf{n}$, resulting in a significant loss in noise immunity.

Let us now consider minimum mean-square error (MMSE) demodulator. The estimation $\hat{\boldsymbol{\theta}}^{\text {MMSE }}$, optimal by MMSE criteria, can be found as follows [2, 4]:

$$
\hat{\theta}^{\mathrm{MMSE}}=\left[\mathrm{H}^{\prime} \mathbf{H}+\mathbf{2} \sigma_{\eta}^{2} \cdot \mathbf{1}\right]^{-1} \mathbf{H}^{\prime} \mathbf{y},
$$

where $\mathbf{2} \boldsymbol{\sigma}_{\boldsymbol{\eta}}^{\mathbf{2}}$ is complex noise dispersion; $\mathbf{1}$ is identity matrix.

Algorithm (3) takes into account the presence of noise in radio channel and, therefore, it has a higher noise immunity compared to ZF algorithm. This can be seen in Figure 2, which shows the dependences of the average BER for ZF and MMSE algorithms for different SNR values. Simulation was performed for MIMO system with 4 transmitting and 4 receiving antennas for 10,000 experiments.

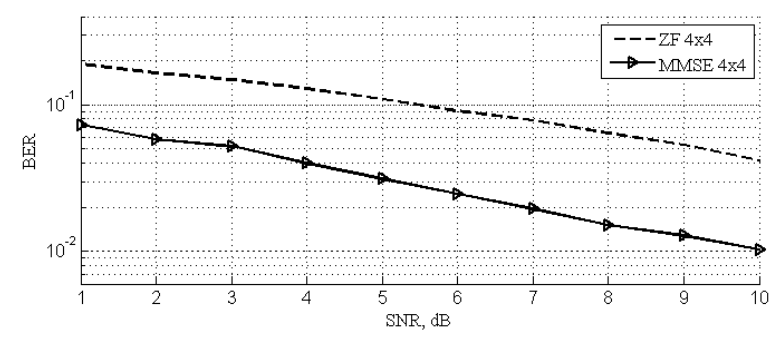

Fig. 2. BER performance for ZF and MMSE demodulation algorithms

To implement the demodulation algorithms considered, it is necessary to calculate the inverse matrix, which is a very difficult task in real-time conditions for systems with large number of antennas. However, it is impossible to calculate in advance the inverse of MIMO channel matrix, which depends on the complex transmission coefficients of radio channel, since matrix $\mathbf{H}$ varies randomly and should be estimated in real-time conditions. Thus, in case of large number of transmitting and receiving antennas, the use of these methods in practice becomes difficult for implementation.

One way to solve this problem is to use iterative methods that have much less computational complexity. These methods iteratively solve a system of linear equations $\mathbf{H} \hat{\mathbf{s}}=\mathbf{y}$, using some initial approximation $\hat{\mathbf{s}}_{\mathbf{0}}$. Approximate solution is consistently calculated during several steps (iterations) [5; 6].

Consider the Chebyshev method. The iteration scheme (4) with variable iteration parameters (5) is called the Chebyshev iteration scheme [5]:

$$
\begin{aligned}
& \frac{\hat{\mathbf{s}}_{i}-\hat{\mathbf{s}}_{i-1}}{\tau_{i}}+\mathbf{H} \hat{\mathbf{s}}_{i-1}=\mathbf{y}, \\
& \gamma_{1} \mathbf{E} \leq \mathbf{H} \leq \gamma_{2} \mathbf{E}, \gamma_{1}>0, \\
& \tau_{i}=\frac{\tau_{0}}{1+\rho_{0} \mu_{i}}, i=1,2, \ldots, n, \tau_{0}=\frac{2}{\gamma_{1}+\gamma_{2}}, \\
& \rho_{0}=\frac{1-\xi}{1+\xi}, \xi=\frac{\gamma_{1}}{\gamma_{2}},
\end{aligned}
$$

where $\gamma_{1}, \gamma_{2}$ are minimum and maximum eigenvalues of matrix $\mathbf{H} ; \mu_{i}=\cos \frac{2 i-1}{2 n} \pi$ is the zeros of the Chebyshev polynomial $\mathrm{T}_{n}(x)=\cos (n \arccos x)$ on the interval $-1 \leq x \leq 1$. In this case, the following estimate takes place:

$$
\left\|\mathbf{H} \hat{\mathbf{s}}_{n}-\mathbf{y}\right\| \leq q_{n}\left\|\mathbf{H} \hat{\mathbf{s}}_{0}-\mathbf{y}\right\|, q_{n} \leq \varepsilon,
$$

where $q_{n}=\frac{2 \rho_{1}^{n}}{1+\rho_{1}^{2 n}}, \quad \rho_{1}=\frac{1-\sqrt{\xi}}{1+\sqrt{\xi}}$. Let us write the expression for the parameters $\tau_{i}$ :

$$
\begin{aligned}
& \tau_{i}=2 /\left[\gamma_{2}+\gamma_{1}+\left(\gamma_{2}-\gamma_{1}\right)\left(\cos \frac{2 i-1}{2 n} \pi\right),\right. \\
& i=1,2, \ldots, n .
\end{aligned}
$$

Let us rewrite the expression (7) of a sequence of iterative parameters with a given maximum number of iterations $i_{\max }$, associated with the roots of Chebyshev polynomials, in the following form [4]:

$$
\tau_{i}=\left[\frac{\gamma_{2}-\gamma_{1}}{2} \cos \left(\frac{i-1 / 2}{i_{\max }} \pi\right)+\frac{\gamma_{2}+\gamma_{1}}{2}\right]^{-1},
$$


where $\gamma_{2}, \gamma_{1}$ are maximum and minimum eigenvalues of the channel matrix; $i_{\max }$ is maximum number of iterations, which is specified in the Chebyshev demodulation algorithm. Table 1 shows the simulation algorithm for the considered iterative demodulator with parameters $\tau_{i}$. The simulation results are the immunity characteristics of the MIMO system for different numbers of antennas.

Table 1

Simulation algorithm for the considered iterative demodulator with parameters $\tau_{i}$

\begin{tabular}{|c|c|c|}
\hline $\begin{array}{c}\text { Step } \\
\text { number }\end{array}$ & $\begin{array}{c}\text { Simulation program } \\
\text { operations }\end{array}$ & $\begin{array}{c}\text { Variables that are } \\
\text { used in the } \\
\text { program }\end{array}$ \\
\hline 1. & $\begin{array}{l}\text { Start of signal-to-noise ratio } \\
\text { cycle }\end{array}$ & SNR \\
\hline 2. & Start of experiments cycle & $L=10000$ \\
\hline \multirow[t]{4}{*}{3.} & Transmitting antennas cycle & $M$ \\
\hline & $\begin{array}{l}\text { Generation of a uniformly } \\
\text { distributed random variable } \\
\text { (for each antenna) }\end{array}$ & $x$ \\
\hline & Bit generation (1 or 0 ) from $x$ & $b$ \\
\hline & $\begin{array}{l}\text { Modulation (generation of } \\
\text { information symbol for each } \\
\text { antenna) }\end{array}$ & $s$ \\
\hline 4. & End of the cycle (step 3 ) & \\
\hline 5. & $\begin{array}{l}\text { Formation of a vector from } \\
\text { generated } \\
\text { symbols for all transmitting } \\
\text { antennas }\end{array}$ & $s$ \\
\hline 6. & $\begin{array}{lr}\text { MIMO channel matrix } \\
\text { generation, } \\
\text { complex } \\
\text { coefficients }\end{array}$ & $\boldsymbol{H}$ \\
\hline 7. & $\begin{array}{l}\text { Generation of complex } \\
\text { Gaussian noise vector }\end{array}$ & $n$ \\
\hline 8. & $\begin{array}{l}\text { Simulation of signal and noise } \\
\text { mixture }\end{array}$ & $\boldsymbol{y}=\boldsymbol{H} \boldsymbol{s}+\boldsymbol{n}$ \\
\hline 9. & $\begin{array}{l}\text { Application of the Chebyshev } \\
\text { method for demodulation }\end{array}$ & \\
\hline 10. & Star of iterations cycle & i_max \\
\hline 11. & $\begin{array}{l}\text { Obtaining estimate on the } i \text {-th } \\
\text { iteration }\end{array}$ & $\hat{\mathbf{S}}_{i}$ \\
\hline 12. & End of cycle (step 10) & \\
\hline 13. & $\begin{array}{l}\text { Demodulation (obtaining } \\
\text { estimate of the received vector } \\
\text { of information symbols) }\end{array}$ & $\hat{\mathbf{S}}$ \\
\hline 14. & $\begin{array}{l}\text { Obtaining estimate of received } \\
\text { bits vector }\end{array}$ & $\hat{\mathbf{b}}$ \\
\hline 15. & $\begin{array}{l}\text { Comparing vectors } \hat{\mathbf{b}} \text { и } \mathbf{b} \text { and } \\
\text { detection of errors }\end{array}$ & errors \\
\hline 16. & Number of errors calculation & sum \\
\hline 17. & End of cycle (step 2) & \\
\hline 18. & $\begin{array}{l}\text { Bit error ratio calculation for } \\
\text { each SNR value }\end{array}$ & $B E R$ \\
\hline 19. & End of cycle (step 1) & \\
\hline
\end{tabular}

\begin{tabular}{l|l}
20. & $\begin{array}{l}\text { Plotting bit error ratio versus } \\
\text { signal-to-noise ratio for } \\
\text { different numbers of antennas }\end{array}$
\end{tabular}

$B E R=f(S N R)$

Figure 3 shows the noise immunity dependencies $B E R=f(S N R)$ obtained by the minimum mean square error (MMSE) method and the Chebyshev method using expression (8) for the parameters $\tau_{i}$ at different signal-to-noise ratio (SNR) values. The simulation was carried out for MIMO system with 4 transmitting and 4 receiving antennas with a number of experiments equal to 10,000 .

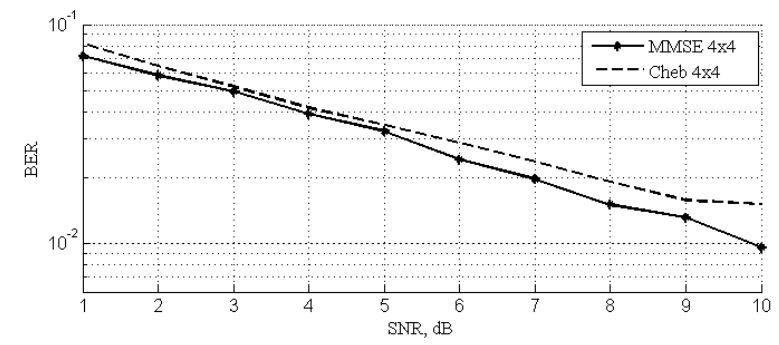

Fig. 3. BER performance of MMSE and Chebyshev demodulation algorithms

For the algorithm (4)-(7) due to computational instability, it is not indifferent in what sequence the zeros of the Chebyshev polynomial are taken. Next, we present a rule for constructing such a sequence of parameters $\left\{\tau_{i}\right\}$ of (8), for which the convergence of the iterative method is monotonic and there is no computational instability. From the sequence, it is necessary to form a permutation containing only the values of the iterative parameters with odd numbers and arrange them in a certain order as follows $[4 ; 5]$ :

$$
\theta_{1}=\{1\}, \theta_{2 i-1}^{(2 m)}=\theta_{i}^{(m)}, \theta_{2 i}^{(2 m)}=4 m-\theta_{2 i-1}^{(2 m)}
$$

Optimal permutation for 8 iterations is the following [5]: $\{1$; $15 ; 7 ; 9 ; 3 ; 13 ; 5 ; 11\}$.

Using the permutations, we can get better convergence with the same number of iterations (Fig. 4).

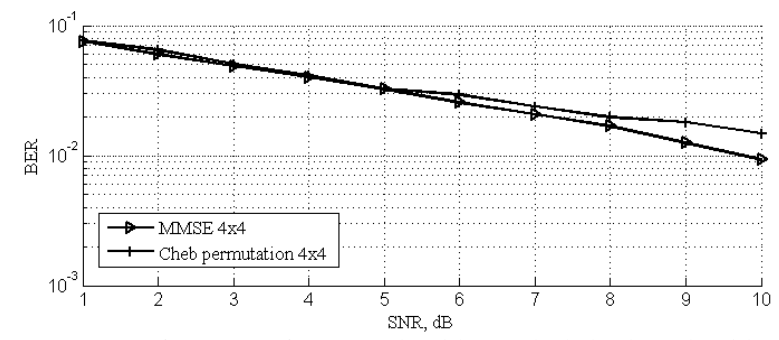

Fig. 4. BER performance of MMSE algorithm and Chebyshev algorithm with permutations of the iterative parameters

The rate of convergence of the iterative process (4)-(7) with the permutation of the iterative parameters in (9) can be found from the following expression [5]:

$$
n=n_{0}(\varepsilon)=\frac{1}{2 \sqrt{\xi}} \ln \frac{2}{\varepsilon},
$$


where $n_{0}(\varepsilon)$ is number of iterations sufficient to solve the system of linear equations with a given accuracy $\varepsilon>0$.

The Chebyshev method provides fast convergence, but its application requires information about the minimum and maximum eigenvalues of the MIMO channel matrix.

Figure 5 shows the dependencies of noise immunity for the MMSE method and the Chebyshev method with permutations of iterative parameters, in which the eigenvalues of matrix $\mathbf{T}$ were used when calculating the iteration parameters:

$$
\mathbf{T}=\mathbf{H}^{\prime} \mathbf{H}+2 \sigma_{\eta}^{2} \cdot \mathbf{1}
$$

where $2 \sigma_{\eta}^{2}$ is complex noise dispersion; $\mathbf{1}$ is identity matrix; $\mathbf{H}^{\prime}$ is Hermitian conjugate matrix with respect to matrix $\mathbf{H}$.

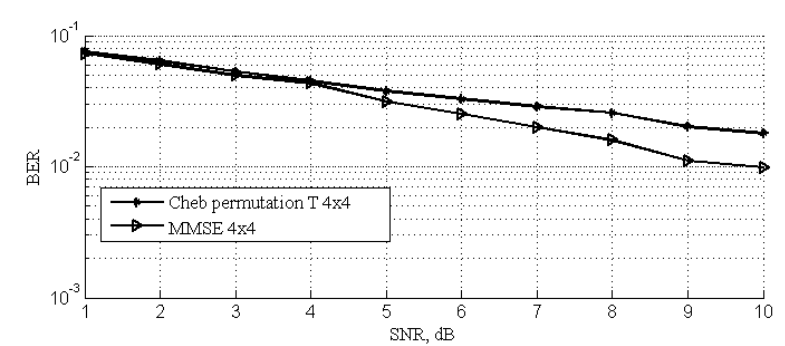

Figure 5. BER performance of MMSE algorithm and Chebyshev algorithm with permutations of the iterative parameters and eigenvalues of matrix $\mathbf{T}$

Massive MIMO technology in future 5G systems [7; 8] will provide a significant increase in capacity of such systems. The considered demodulation algorithm based on the Chebyshev method is planned to be used for MIMO systems with large number of antennas, as well as for signals with higher modulation order. The main limitation of Chebyshev iteration method applicability is the need for a priori knowledge of MIMO channel matrix $\mathbf{H}$ spectrum boundaries (maximum and minimum eigenvalues), and with decreasing accuracy of these values, the convergence of the algorithm slows down. The task of finding and applying estimates of MIMO channel matrix eigenvalues in demodulation algorithms requires additional research.

\section{REFERENCES}

[1] Wei Xiang, Kan Zheng, Xuemin (Sherman) Shen. 5G Mobile Communications. [S.1.]: Springer International Publishing, 2017.

[2] Бакулин М.Г., Варукина Л.А., Крейнделин В.Б. Технология MIMO: принципы и алгоритмы. М.: Горячая линия - Телеком, 2014 [Bakulin M.G., Varukina L.A., Kreyndelin V.B. MIMO Technology: Principles and Algorithms. Moscow: HotlineTelecom, 2014].

[3] Панкратов Д.Ю., Степанова А.Г. (МТУСИ, Москва) Моделирование системы МIMO // Международная научнотехническая конференция "INTERMATIC-2017". Ноябрь 2017. M.: МИРЕA, 2017. С. 1052-1056. [Pankratov D.Yu., Stepanova A.G. MIMO system simulation // Proceedings of the International Scientific and Technical Conference "INTERMATIC-2017", November, 2017. Moscow: MIREA, 2017. P. 1052-1056.

[4] Бакулин М.Г., Крейнделин В.Б., Панкратов Д.Ю. Технологии в системах радиосвязи на пути к 5G. М.: Горячая линия Телеком, 2018. [Bakulin M.G., Kreyndelin V.B., Pankratov D.Yu. Technologies in Radio Communication Systems on the Way to 5G. Moscow: Hotline-Telecom, 2018].

[5] Самарский А.А. Теория разностных систем. М.: Наука, 1977. [Samarskii A.A. Theory of Difference Schemes. Moscow: Nauka, 1977],

[6] Шлома А.М., Бакулин М.Г., Крейнделин В.Б. Новые алгоритмы формирования и обработки сигналов в системах подвижной связи / Под ред.А.М. Шломы. М.: Горячая линия - Телеком, 2008. [Shloma A. M., Bakulin M. G., Kreyndelin V. B. et al. New Algorithms for Generating and Processing signals in Mobile Communication Systems / Ed. by prof. A.M. Shloma. Moscow: Hotline-Telecom, 2008].

[7] Luo F.-L., Zhang C. Signal processing for 5G: algorithms and implementations. Chichester,: John Wiley \& Sons Inc., 2016.

[8] Wunder G., Jung P., Wiedmann F. 5G NOW: Non-orthogonal, Asynchronous Waveforms for Future Mobile Applications // IEEE Communications Magazine. 2014. Vol. 52. N 2. 